PROCEEDINGS OF THE

AMERICAN MATHEMATICAL SOCIETY

Volume 138, Number 3, March 2010, Pages 1153-1157

S 0002-9939(09)10137-5

Article electronically published on October 28, 2009

\title{
ON EMBEDDING THE INFINITE CYCLIC COVERINGS OF KNOT COMPLEMENTS INTO THREE SPHERE
}

\author{
ZHIQING YANG \\ (Communicated by Alexander N. Dranishnikov)
}

\begin{abstract}
We construct a class of knots with the CI* property, that is, $\pi_{1}(M(n) \mid \partial M(n)) \neq\{e\}$ for some $n>0$. It follows that the infinite cyclic covering of such a knot cannot be embedded in any compact 3-manifold.
\end{abstract}

\section{INTRODUCTION}

A (tame) knot is a smooth (or PL) embedding $f: S^{1} \rightarrow S^{3}$. Let $E(k)=$ $S^{3}-N(k)$ denote the compact complement of a knot $k$. Let $\widetilde{E}(k)$ denote the infinite cyclic covering of $E(k)$ [4. A natural question is:

\section{QUESTION}

When does $\widetilde{E}(k)$ embed in $S^{3}$ ?

Professor Robert D. Edwards attributes this problem to J. Stallings. In 2, Jiang, $\mathrm{Ni}$, Wang and Zhou studied the above problem. They gave a necessary condition for the existence of such an embedding when the genus equals one. Later, C. McA. Gordon [1] showed that the infinite cyclic cover of the exterior of the untwisted Whitehead double of a non-trivial knot does not embed in any compact 3-manifold. In this paper, we describe a class of knots whose infinite cyclic covers do not embed in any compact 3-manifold. We approach the question from a group-theoretical point of view and construct a class of knots with the CI* property (section 2). The infinite cyclic covering of a $\mathrm{CI}^{*}$ knot does not embed in any compact 3-manifold. On the other hand, $9_{46}$ is a non-fibered, non-CI* knot by [2].

\section{The MAin EXAMPLE}

Let $k$ be a knot in $S^{3}$. A Seifert surface of $k$ is a once punctured orientable surface of genus $g$, which we regard as a disk with $2 g$ bands glued to it. Figure 1 is a punctured torus. Figure 2 is another way to denote it. We use lines to indicate the bands. If there are some twists in a band, we will indicate the twists in the text.

Received by the editors July 26, 2008, and, in revised form, February 26, 2009, July 17, 2009, and July 29, 2009.

2010 Mathematics Subject Classification. Primary 57M25; Secondary 57M05.

Key words and phrases. infinite cyclic covering, knot, Seifert surface, knot group.

The author is supported by a grant (No. 100771023) of NSFC and a grant from Specialized Research Fund for the Doctoral Program of Higher Education (SRFDP) (20070141035).

(C)2009 American Mathematical Society Reverts to public domain 28 years from publication 


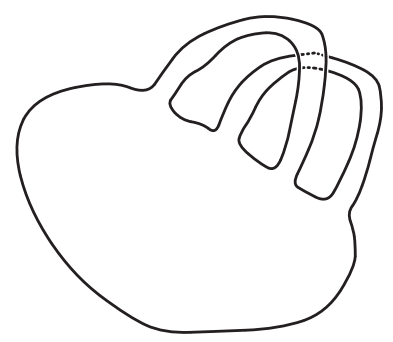

Figure 1. A punctured torus

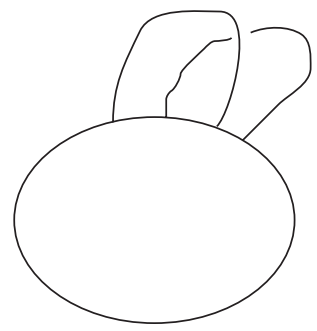

FiguRE 2. Another picture of the punctured torus

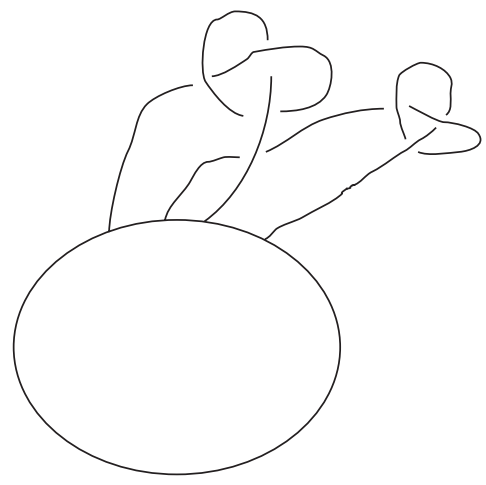

Figure 3 . The main example

In this paper, from now on, we do not draw those bands; we just use the second type figures. Figure 3 is our primary example. Here, the core of each band forms a part of a trefoil knot, and along each band there are three positive full twists. The picture shows a genus 1 Seifert surface $F$ with boundary a knot $k$. We orient the surface $F$ in such a way that the up side of the disk is positive. Later, we shall tell how this can be generalized.

Lemma 2.1. The fundamental group $G$ of $S^{3}-F$ is a free product $G=H * H$, where $H$ is the fundamental group of the trefoil knot complement.

Proof. If we regard the disk as a 3 -ball, then the space $S^{3}-F$ can be deformed into a union of two trefoil knot complements attached along a 2 dimensional disk.

Remark 2.2. $H$ has the presentation: $\left\langle x_{1}, x_{2}, x_{3} \mid x_{1} x_{2}=x_{2} x_{3}=x_{3} x_{1}\right\rangle$. 
Definition 2.3. Let $N$ be a manifold with boundary, and let $A$ be a subset of $N$. We use $\pi_{1}(N \mid A)$ to denote the group $\pi_{1}(N) /\left\langle i_{*}\left(\pi_{1}(A)\right)\right\rangle^{n}$, where $\left\langle i_{*}(\pi(A))\right\rangle^{n}$ denotes the normal closure of the group $i_{*}(\pi(A))$ in $\pi_{1}(N)$, and $i: A \rightarrow N$ is the inclusion map.

Let $F$ be a Seifert surface of a knot $k$. If we cut $E(k)$ along $F$, we will get a 3-manifold $M$ with a connected boundary which is a union of two copies $F^{+}, F^{-}$ of $F$ and an annulus $A$. The infinite cyclic covering $\widetilde{E}(k)$ can be constructed by taking a countably infinite collection of copies of $M$ and identifying $F^{+}$in the $i$ th copy with $F^{-}$in the $(i+1)$ st copy for each $i$. Let $M(n)$ be the submanifold of $\widetilde{E}(k)$ consisting of $n$ consecutive copies of $M$. Thus the boundary of $M(n)$ is also a union of two copies $F^{+}(n), F^{-}(n)$ of $F$ and an annulus $A$.

Lemma 2.4. For the knot $k$ illustrated above, the image of $i_{*}: \pi_{1}\left(F^{-}\right) \rightarrow \pi_{1}(M \mid$ $\left.F^{+}\right)$is a rank two free abelian group.

Proof. The fundamental group $G$ of $M$ is a free product $H * H$, and $H$ has the presentation: $\left\langle x_{1}, x_{2}, x_{3} \mid x_{1} x_{2}=x_{2} x_{3}=x_{3} x_{1}\right\rangle$. We denote the two copies of $H$ by $H_{1}, H_{2}$. Hence $G$ has the presentation:

$$
H_{1} * H_{2}=\left\langle x_{1}, x_{2}, x_{3}, y_{1}, y_{2}, y_{3} \mid x_{1} x_{2}=x_{2} x_{3}=x_{3} x_{1}, y_{1} y_{2}=y_{2} y_{3}=y_{3} y_{1}\right\rangle .
$$

The group $i_{*}\left(\pi_{1}\left(F_{1}^{+}\right)\right)$is normally generated by $a_{1}^{+}, a_{2}^{+}$which are represented by the simple closed curves that are the cores of two bands. The group $i_{*}\left(\pi_{1}\left(F_{1}^{-}\right)\right)$ is generated by the same curves $a_{1}^{-}, a_{2}^{-}$pushed to the negative side of the Seifert surface. We can check the following relations:

$a_{1}^{+} y_{1}^{-1}=a_{1}^{-}, a_{2}^{-} x_{1}^{-1}=a_{2}^{+}$.

Since we only work on the normal closure of $i_{*}\left(\pi_{1}\left(F_{1}^{+}\right)\right)$, then, up to some base point change, we can take $a_{1}^{+}=x_{2} x_{1} x_{3}=x_{2} x_{1} x_{2}^{-1} x_{1} x_{2} x_{1}^{-3}, a_{2}^{+}=$ $y_{2} y_{1} y_{2}^{-1} y_{1} y_{2} y_{1}^{-3} x_{1}^{-1}$. Let $\alpha: H_{1} \rightarrow Z$ denote the abelianization map, then $\alpha\left(x_{i}\right)=$ 1 , and $\alpha\left(a_{1}^{+}\right)=0$. Hence in the group $H_{1} /\left\langle a_{1}^{+}\right\rangle^{n}$, each $x_{i}$ is of infinite order. $\pi_{1}\left(M \mid F^{+}\right)=G /\left\langle a_{1}^{+}, a_{2}^{+}\right\rangle^{n}$ can be rewritten as a free product with amalgamation

$$
\left(H_{1} /\left\langle a_{1}^{+}\right\rangle^{n}\right) *_{x_{1}=y_{2} y_{1} y_{2}^{-1} y_{1} y_{2} y_{1}^{-3}} H_{2} .
$$

The image $i_{*}\left(\pi_{1}\left(F_{1}^{-}\right)\right)$is generated by $\left\{a_{1}^{-}, a_{2}^{-}\right\} \sim\left\{y_{1}^{-1}, x_{1}\right\} \sim\left\{x_{1}, y_{1}\right\} \sim$ $\left\{y_{2} y_{1} y_{2}^{-1} y_{1} y_{2} y_{1}^{-3}, y_{1}\right\}$. This is the meridian and longitude pair in a trefoil knot group $H_{2}$. Hence the subgroup generated by $\left\{y_{2} y_{1} y_{2}^{-1} y_{1} y_{2} y_{1}^{-3}, y_{1}\right\}$ in $H_{2}$ is a rank two free abelian group.

By the theorems on group amalgamation [3], $H_{2}$ embeds in $\pi_{1}\left(M \mid F^{+}\right)$; hence the image of $i_{*}: \pi_{1}\left(F^{-}\right) \rightarrow \pi_{1}\left(M \mid F^{+}\right)$is a rank two free abelian group.

Remark 2.5. Likewise, the image of $i_{*}: \pi_{1}\left(F^{+}\right) \rightarrow \pi_{1}\left(M \mid F^{-}\right)$is a rank two free abelian group.

Lemma 2.6. $\pi_{1}(M(2) \mid \partial M(2)) \neq\{e\}$.

Proof. $M(2)$ is a union of 2 copies of $M$, say $M_{1}$ and $M_{2}$, glued along $F_{1}^{+}$and $F_{2}^{-}$. By van Kampen's theorem, and by the previous lemma, $\pi_{1}(M(2) \mid \partial M(2))$ is a free product of $\pi_{1}\left(M \mid F^{-}\right)$and $\pi_{1}\left(M \mid F^{+}\right)$amalgamated along a rank two free abelian group. Hence it is non-trivial.

Proposition 2.7. (1) $M(2)$ does not embed in $S^{3}$.

(2) For any compact 3-manifold $N$, if $n$ is sufficiently large, $M(2 n)$ does not embed in $N$. 
Proof. (1) If $M(2)$ embeds in $S^{3}$, let $A$ denote $S^{3}-M(2)$. Then, by van Kampen's theorem, $\{e\}=\pi_{1}\left(S^{3} \mid A\right)=\pi_{1}(M(2) \mid \partial M(2))$. This contradicts Lemma 3.

(2) There is a surjective homomorphism from $\pi_{1}(M(2 n) \mid \partial M(2 n))$ onto $*_{n}\left(\pi_{1}(M(2) \mid \partial M(2))\right)$. For any compact 3-manifold $N$, if $M(2 n)$ embeds into $N$, then by van Kampen's theorem, there is a surjective homomorphism from $\pi_{1}(N)$ onto $\pi_{1}(N \mid N-M(2 n)) \cong \pi_{1}(M(2 n) \mid \partial M(2 n))$, and hence onto $*_{n}\left(\pi_{1}(M(2) \mid\right.$ $\partial M(2)))$. For a fixed $N, n$ can't be arbitrarily large. This is a contradiction.

Definition 2.8. 1 . We say that a knot has property $\mathrm{CI}^{*}$, or that it is a $\mathrm{CI}^{*}$ knot, if $\pi_{1}(M(n) \mid \partial M(n)) \neq\{e\}$ for some $n>0$.

2. We say that a knot has property CI, or that it is a CI knot, if the commutator subgroup of the knot group has $K^{\infty}$ (the free product of countable many copies of some group $K \neq\{e\})$ as a quotient.

Denote the homomorphism as $p_{1}: G^{\prime}(k) \rightarrow K^{\infty}$, where $G^{\prime}(k)$ is the commutator subgroup of the knot group.

Theorem 2.9. (1) There exist CI* knots.

(2) The infinite cyclic covering of a $C I^{*}$ knot does not embed in any compact 3-manifolds.

Proposition 2.10. 1. The CI* property does not depend on the choice of the Seifert surface.

2. A CI* knot $k$ is clearly a CI knot.

3. If we assume the following hypothesis, then any CI knot is a CI* knot too.

Hypothesis. Let $g$ be a positive integer, and let $K^{l}$ be the free product of $l$ copies of any non-trivial group $K$. Then if $l$ is big enough, any quotient group of $K^{l}$ by adding $g$ relations is a non-trivial group.

Proof of Proposition 2.10(1). Choose any two Seifert surfaces $F, F^{\prime}$ for a knot $k$ and construct the two spaces $M(n)$ and $M^{\prime}(m)$. For any $m>0$, if $n$ is big enough, then $M^{\prime}(m)$ embeds in $M(n)$. It follows that if $\pi_{1}\left(M^{\prime}(m) \mid \partial M^{\prime}(m)\right) \neq\{e\}$, then $\pi_{1}(M(n) \mid \partial M(n)) \neq\{e\}$.

Proof of Proposition 2.10(3). Suppose $k$ is a CI knot. We have the following commutative diagram:

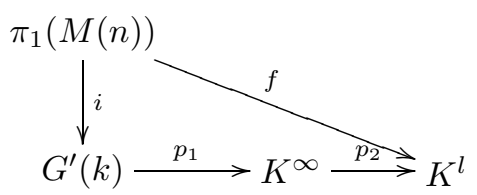

Here, $G^{\prime}(k) \cong \pi_{1}(\widetilde{E}(k)), i$ is induced by the inclusion map, $p_{2}$ is a projection to finitely many factors, $f$ is the composition of those homomorphisms. $p_{1}$ and $p_{2}$ are surjective, and for any $l$, if we choose $n$ big enough, $f$ will be surjective too.

$\partial M(n)$ is a surface of fixed genus, say $g$. If $\pi_{1}(M(n) \mid \partial M(n))=\{e\}$ for any $n>0$, then $\pi_{1}(M(n))$ is normally generated by $2 g$ elements. This contradicts our hypothesis on groups if $l$ is big enough. 


\section{DisCUSSION AND GENERALIZATIONS}

The reader might already have noticed that our example can be extensively generalized. First of all, the core of the two bands can be other knots, and the twisting of the bands can vary too. Secondly, one can add more bands to construct higher genus knots.

On the other hand, fibered knots are clearly not CI*. Their infinite cyclic coverings embed in $S^{3}$. There are other knots, like $9_{46}$, 2, which are non-fibered but whose infinite cyclic coverings embed in $S^{3}$. Those knots are called IE knots (infinite cyclic covering embeds in $S^{3}$ ). Therefore

$\{$ fibered knots $\} \subset\{$ IE knots $\} \subset\{$ non-CI* knots $\}$

If $k$ is a non-CI* knot, then $\pi_{1}(M(n) \mid \partial M(n))=\{e\}$ for any $n>0$. This suggests that $M(n)$ most likely embeds in $S^{3}$. We propose the following conjectures.

Conjecture. 1. Non-CI* knots are IE knots.

2. Property $C I$ and property $C I^{*}$ are equivalent.

\section{ACKNOWLEDGEMENTS}

The author would like to thank Professor Shicheng Wang for introducing him to this interesting problem, and Professor Ruifeng Qiu for helpful discussions.

\section{REFERENCES}

1. C. McA. Gordon, On embedding infinite cyclic covers in compact 3-manifolds, preprint, Math. GT/0608339, http://front.math.ucdavis.edu/0608.5339.

2. Boju Jiang, Yi Ni, Shicheng Wang and Qing Zhou, Embedding infinite cyclic covers of knot spaces into 3-space, Topology, vol. 45, issue 4, July 2006, 691-705. MR.2236373 (2007g:57010)

3. W. Magnus, A. Karrass and D. Solitar, Combinatorial group theory, Wiley, 1966 (reprinted by Dover, 1976). MR0422434 (54:10423)

4. D. Rolfsen, Knots and links, Publish or Perish Inc., Berkeley, 1976, 160-197. MR0515288 $(58: 24236)$

School of Mathematical Science, Dalian University of Technology, Dalian, Liaoning 116024, People's Republic of China

E-mail address: yangzhq@dlut.edu.cn 